\title{
COVID-19 in Gaza: a pandemic spreading in a place already under protracted lockdown
}

\author{
Osaid Alser, ${ }^{1}$ Shaymaa AlWaheidi, ${ }^{2}$ Khamis Elessi ${ }^{3}$ and Hamza Meghari ${ }^{4}$
}

${ }^{1}$ Massachusetts General Hospital, Harvard Medical School, Boston, United States of America. ${ }^{2}$ King's College London, London, United Kingdom. ${ }^{3}$ Faculty of Medicine, Islamic University of Gaza, Gaza, Palestine. ${ }^{4}$ University College London, London, United Kingdom. (Correspondence to: Osaid Alser: osaidalserr@hotmail.com).

Citation: Alser O; AlWaheidi S; Elessi K; Meghari H. COVID-19 in Gaza: a pandemic spreading in a place already under protracted lockdown. East Mediterr Health J. 2020;26(7):762-763 https://doi.org/10.26719/emhj.20.089

Received: 23/05/20; accepted: 02/07/20

Copyright (C) World Health Organization (WHO) 2020. Open Access. Some rights reserved. This work is available under the CC BY-NC-SA 3.0 IGO license (https://creativecommons.org/licenses/by-nc-sa/3.o/igo)

Gaza is a densely populated area that forms part of the Occupied Palestinian Territories, and inhabited by approximately 2 million Palestinians of whom the majority are registered refugees living in overcrowded camps (1). The prolonged blockade imposed since 2007 has severely impacted the socioeconomic and health conditions of Gazans (2). The longstanding movement restrictions have undermined Gaza's economy, resulting in high levels of unemployment, food insecurity, aid dependency and poor standards of hygiene and sanitation. The Palestinian Ministry of Health swiftly declared a state of emergency in early March 2020 following the report of 5 cases of COVID-19 in Gaza, and subsequently shifted public health efforts and medical supplies to respond to the emergency. To prevent the spread of COVID-19, both the Israeli and Palestinian authorities in Gaza and the West Bank now request that patients do not leave Gaza, except for emergency cases and certain cancer patients.

However, these new movement restrictions are increasing inequity in access to health care for patients with noncommunicable diseases, particularly cancer, as well as other vulnerable groups. This policy so far has caused a $40 \%$ reduction in the number of medical referrals for patients in Gaza (901 referrals from Gaza in May 2020 compared to an average 2000 referrals per month in the first quarter of 2020). Moreover, a $5 \%$ decline in Israeli travel permit approval rates for referred patients has been reported in May 2020 compared with the first quarter of 2020 (3). The Palestinian Government in Gaza has also discouraged travel permit applications that could be delayed, and the compulsory 21-day quarantine for everyone entering or re-entering Gaza has been a further deterrent to patients applying for treatments outside of Gaza.

Nearly 4000 elective surgeries have been postponed due to the COVID-19 preparedness and response plan (4). Secondary cancer prevention programmes have also been interrupted, and in the coming months this could mean that delays in cancer diagnosis in Gaza may become even more common. In addition, Several major challenges are increasing the threat of COVID-19 infection in Gaza, including 1) overstretched health-care system; 2) inadequate access to safe water, sanitation and hygiene (WASH) services, which are essential to infection prevention; and 3) high density of population, especially in refugee camps where self-isolation is almost impossible (5). Currently, there are only 63 adult intensive care unit beds with ventilators in governmental hospitals in Gaza, which are barely enough for routine cases, along with a limited number of available donated personal protective equipment (PPE) $(6,7)$. Nearly half of the essential drugs list, including cancer chemotherapy, are at a zero stock levels (8).

As of June 11, 2020, there have been 72 confirmed cases of COVID-19 in Gaza with a case fatality rate (CFR) of $0.81 \%$. These figures appear very low compared to elsewhere in the Occupied Palestinian Territories (547 cases in the West Bank including East Jerusalem) and the rest of the world (Figure 1) (6)

However, the numbers are expected to rise exponentially and therefore it is imperative to start thinking strategically of what can realistically be done before losing control over the current situation. Unlike the 'one size fits all' approach, measures that have proven successful in other countries might not be effective in densely populated and disadvantaged environments such as Gaza. Avoiding social gatherings or observing the two-metre distancing measure could well be viewed as foreign concepts and its effectiveness will be limited among Palestinian extended families living in overcrowded refugee camps.

With this in mind, proactive and proven public health interventions of 'test, trace and isolate' must be implanted at a large-scale. So far, and despite international funding, there continues to be a very limited testing capacity in Gaza due to the lack of laboratory testing kits. Nevertheless, strict safety regulations, including a mandatory 21-day home quarantine for all people coming through the two passenger crossings with Israel (Erez) and Egypt (Rafah), have significantly reduced the virus transmission to the population in Gaza. The Palestinian Authority in Gaza has agreed with Egypt to keep the Rafah crossing closed in both directions since May 15, and only allow occasional return to Gaza on a case-by-case 
Figure 1 Geographical locations of COVID-19 in the Occupied Palestinian Territories (adapted from the WHO Situation Report 32) (6)

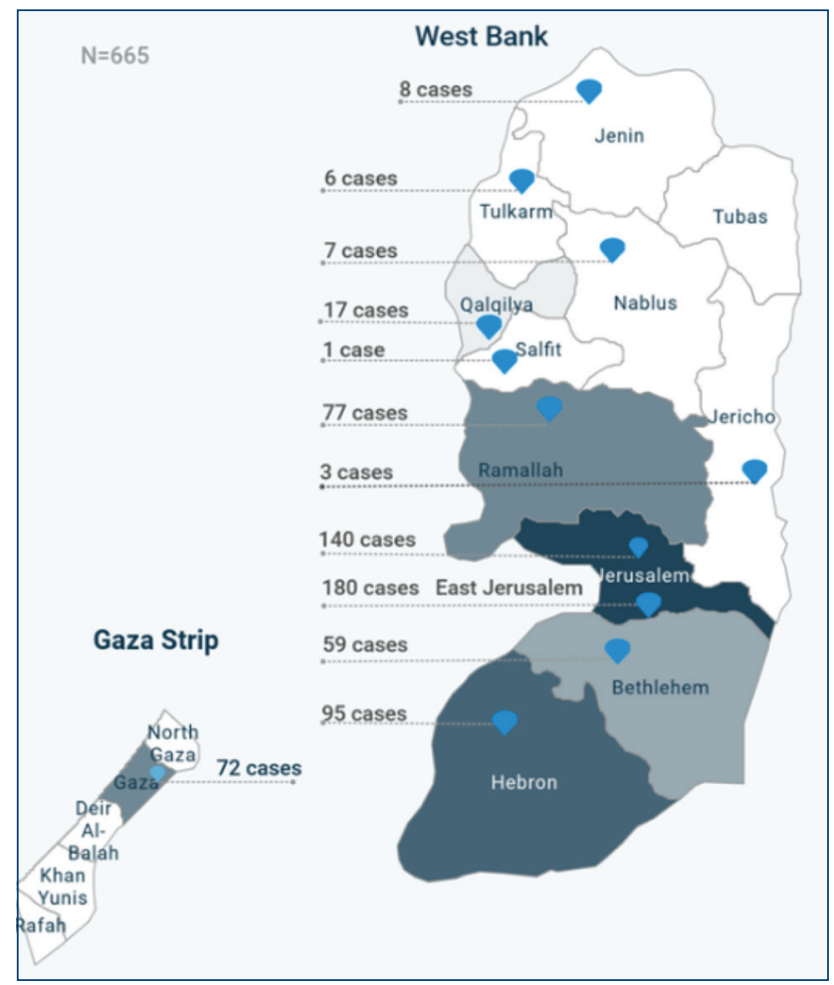

basis in order to regulate the capacity in their quarantine facilities. Access to health care in and out of Gaza through the Erez crossing, which has been extremely limited due to COVID-19, is now stopped by the decision of the Gazan Authority to suspend security coordination in response to Israel's annexation plans.

COVID-19 has resulted in high levels of uncertainty, anxiety, social isolation, and further inequity in health care for Palestinians in Gaza. Thus, it is important to maintain the availability and access to essential health care and WASH services, and to scale up testing and tracing capacities, all of which are needed during this period to reduce potential health consequences. There may be no way to prevent a COVID-19 outbreak in Gaza, but whenever COVID-19 treatments or vaccines become available, equal allocation to Gaza and other disadvantaged environments might be problematic. Meanwhile, it is essential to focus efforts on testing, tracing and isolating, awareness and advocacy interventions about the disease, immediate access to handwashing, more training in infection prevention and control for Palestinian healthcare workers, more compliance to mitigation procedures, as well as improved access to health care for chronically ill patients in Gaza.

\section{References}

1. Palestinian Central Bureau of Statistics (PCBS). About 13 million Palestinians in the historical Palestine and Diaspora. Ramallah: PCBS, 2019 (http://www.pcbs.gov.ps/post.aspx?lang=en\&ItemID=3503, accessed May 18, 2020).

2. The United Nations Relief and Works Agency for Palestine Refugees in the Near East (UNRWA). Gaza Strip. Amman: UNRWA; 2020 (https://www.unrwa.org/where-we-work/gaza-strip, accessed May 18, 2020).

3. World Health Organization Regional Office for the Eastern Mediterranean (WHO/EMRO). Health access monthly report March 2020 - Health Access Barriers for patients in the occupied Palestinian territory. Cairo: WHO/EMRO; 2020 (http://www.emro. who.int/images/stories/palestine/documents/March_2020_Monthly.pdf?ua=1, accessed May 3, 2020).

4. World Health Organization Regional Office for the Eastern Mediterranean (WHO/EMRO). Health Cluster March Report. The occupied Palestinian territory. Cairo: WHO/EMRO; 2020 (http:/www.emro.who.int/images/stories/palestine/documents/ Health_Cluster-Bulletin-Marcho4_March31.pdf?ua=1, accessed May 3 2020).

5. State of Emergency: Palestine's COVID-19 Response Plan. Ramallah: Palestinian Authority; 2020 (https://reliefweb.int/report/ occupied-palestinian-territory/state-emergency-palestine-s-covid-19-response-plan, accessed May 18, 2020).

6. World Health Organization Regional Office for the Eastern Mediterranean (WHO/EMRO). Coronavirus disease (COVID-19) Situation Update 32, issued June 11, 2020. The occupied Palestinian territory. Cairo: WHO/EMRO; 2020 (https://who18. createsend.com/campaigns/reports/viewCampaign.aspx?d=j\&c=99FA4938Do49E3A8\&ID=B2DB34806A7129562540EF23F30FED ED\&temp=False\&tx=o\&source=Report\&fbclid=IwAR3JBcFUP7-ooUgmAiqnEgU-Jtw3dZ94OtEY_-gKePD9tGozUmcdolM2mHQ, accessed June 23, 2020).

7. Alser O, Alghoul H, Alkhateeb Z, Hamdan A, Albarqouni L, Saini K. Healthcare workers preparedness for COVID-19 pandemic in the occupied Palestinian territory: a cross-sectional survey. medRxiv 2020: (https://www.medrxiv.org/ content/10.1101/2020.05.09.20096099V2) DOI: 10.1101/2020.05.09.20096099.

8. World Health Organization Regional Office for the Eastern Mediterranean (WHO/EMRO). Situation report - occupied Palestinian territory, Gaza, o -31 August 2019. Cairo: WHO/EMRO; 2019 (http://www.emro.who.int/images/stories/palestine/ documents/sitrep_aug_2019_vo_sh_rev_gro.pdf?ua=1). 